\title{
Individuals with mutations in XPNPEP3, which encodes a mitochondrial protein, develop a nephronophthisis-like nephropathy
}

John F. O'Toole, ${ }^{1}$ Yangjian Liu, ${ }^{2}$ Erica E. Davis, ${ }^{2,3}$ Christopher J. Westlake, ${ }^{4}$ Massimo Attanasio, ${ }^{1}$

Edgar A. Otto, ${ }^{1}$ Dominik Seelow, ${ }^{5,6}$ Gudrun Nurnberg, ${ }^{5}$ Christian Becker, ${ }^{5}$ Matti Nuutinen, ${ }^{7}$ Mikko Kärppä, ${ }^{7}$ Jaakko Ignatius, ${ }^{7}$ Johanna Uusimaa, ${ }^{7}$ Salla Pakanen, ${ }^{7}$ Elisa Jaakkola, ${ }^{7}$ Lambertus P. van den Heuvel, ${ }^{8}$ Henry Fehrenbach, ${ }^{9}$ Roger Wiggins, ${ }^{10}$ Meera Goyal, ${ }^{10}$ Weibin Zhou, ${ }^{1}$ Matthias T.F. Wolf, ${ }^{1}$ Eric Wise, ${ }^{1}$ Juliana Helou, ${ }^{1}$ Susan J. Allen, ${ }^{1}$

Carlos A. Murga-Zamalloa, ${ }^{11}$ Shazia Ashraf,1 Moumita Chaki,1 Saskia Heeringa, ${ }^{1}$ Gil Chernin, ${ }^{1}$ Bethan E. Hoskins, ${ }^{1}$ Hassan Chaib, ${ }^{1}$ Joseph Gleeson, ${ }^{12}$ Takehiro Kusakabe, ${ }^{13,14}$ Takako Suzuki, ${ }^{13,14}$

R. Elwyn Isaac, ${ }^{15}$ Lynne M. Quarmby, ${ }^{16}$ Bryan Tennant, ${ }^{16}$ Hisashi Fujioka, ${ }^{17}$ Hannu Tuominen, ${ }^{18}$ IImo Hassinen, ${ }^{19}$ Hellevi Lohi, 20 Judith L. van Houten, ${ }^{21}$ Agnes Rotig,, ${ }^{22}$ John A. Sayer,1,23 Boris Rolinski, ${ }^{24}$ Peter Freisinger, ${ }^{24}$ Sethu M. Madhavan, ${ }^{25}$ Martina Herzer, ${ }^{26}$

Florence Madignier, ${ }^{26}$ Holger Prokisch,,26,27 Peter Nurnberg, ${ }^{5,28}$ Peter Jackson, ${ }^{4}$ Hemant Khanna, ${ }^{11}$ Nicholas Katsanis,, 2,3,29,30 and Friedhelm Hildebrandt1,31,32

1Department of Pediatrics, University of Michigan, Ann Arbor. ${ }^{2}$ McKusick-Nathans Institute of Genetic Medicine, Johns Hopkins University School of Medicine, Baltimore, Maryland. ${ }^{3}$ Center for Human Disease Modeling, Department of Cell Biology, Duke University Medical Center, Durham, North Carolina. ${ }^{4}$ Genentech Inc., South San Francisco, California. ${ }^{5}$ Cologne Center for Genomics and Institute for Genetics, University of Cologne, Germany.

${ }^{6}$ Department of Neuropediatrics, Charite - Universitaetsmedizin Berlin, Germany. ${ }^{7}$ Department of Pediatrics and Adolescents, Department of Clinical Genetics, and Department of Neurology, Oulu University Hospital, Finland. ${ }^{8}$ Department of Pediatrics, Nijmegen Centre for Mitochondrial Disorders,

Radboud University Nijmegen Medical Centre, Netherlands. ${ }^{9}$ Kinderklinik Memmingen, Germany. ${ }^{10}$ Department of Internal Medicine,

${ }^{11}$ Department of Ophthalmology and Visual Sciences, W.K. Kellogg Eye Center, University of Michigan. ${ }^{12 H o w a r d ~ H u g h e s ~ M e d i c a l ~ I n s t i t u t e, ~}$

Department of Neurosciences, University of California at San Diego, La Jolla. ${ }^{13}$ Department of Life Science, Graduate School of Life Science, University of Hyogo, Japan. ${ }^{14}$ Department of Biology, Faculty of Science and Engineering, Konan University, Kobe, Japan.

15Institute of Integrative and Comparative Biology, Faculty of Biological Sciences, University of Leeds, United Kingdom.

${ }^{16}$ Department of Molecular Biology and Biochemistry, Simon Fraser University, Burnaby, Canada. ${ }^{17}$ Department of Pharmacology,

Case Western Reserve University, Cleveland, Ohio. ${ }^{18}$ Department of Pathology and ${ }^{19}$ Department of Medical Biochemistry, University of Oulu, Finland.

${ }^{20}$ Department of Internal Medicine, Lappland Central Hospital, Rovaniemi, Finland. ${ }^{21}$ Department of Biology and Vermont Chemosensory Group, University of Vermont, Burlington. ${ }^{22}$ Département de Génétique, Université Paris Descartes, Unité INSERM U781, Hôpital Necker-Enfants Malades, France.

${ }^{23}$ Institute of Human Genetics, Newcastle University, Newcastle upon Tyne, United Kingdom. ${ }^{24}$ Academic Hospital Munchen-Schwabing,

Institute for Clinical Chemistry and Metabolic Disease Center, and Department of Pediatrics, Technical University Munich, Germany. ${ }^{25}$ Department of Medicine, MetroHealth Medical System, Cleveland, Ohio. ${ }^{26}$ Institute of Human Genetics, Helmholtz Zentrum Munich, German Research Center for Environmental Health, Neuherberg, Germany. ${ }^{27}$ Institute of Human Genetics, Klinikum rechts der Isar, Technical University Munich, Germany.

${ }^{28}$ Center for Molecular Medicine and Cologne Excellence Cluster on Cellular Stress Responses in Aging-associated Diseases, University of Cologne. ${ }^{29}$ Wilmer Eye Institute and ${ }^{30}$ Department of Molecular Biology and Genetics, Johns Hopkins University School of Medicine. ${ }^{31} \mathrm{Human}$ Genetics and ${ }^{32} \mathrm{Howard}$ Hughes Medical Institute, University of Michigan.

The autosomal recessive kidney disease nephronophthisis (NPHP) constitutes the most frequent genetic cause of terminal renal failure in the first 3 decades of life. Ten causative genes (NPHP1-NPHP9 and NPHP11), whose products localize to the primary cilia-centrosome complex, support the unifying concept that cystic kidney diseases are "ciliopathies". Using genome-wide homozygosity mapping, we report here what we believe to be a new locus (NPHP-like 1 [NPHPL1]) for an NPHP-like nephropathy. In 2 families with an NPHP-like phenotype, we detected homozygous frameshift and splice-site mutations, respectively, in the $X$-prolyl aminopeptidase 3 (XPNPEP3) gene. In contrast to all known NPHP proteins, XPNPEP3 localizes to mitochondria of renal cells. However, in vivo analyses also revealed a likely cilia-related function; suppression of zebrafish $x p n p e p 3$ phenocopied the developmental phenotypes of ciliopathy morphants, and this effect was rescued by human XPNPEP3 that was devoid of a mitochondrial localization signal. Consistent with a role for XPNPEP3 in ciliary function, several ciliary cystogenic proteins were found to be XPNPEP3 substrates, for which resistance to $\mathrm{N}$-terminal proline cleavage resulted in attenuated protein function in vivo in zebrafish. Our data highlight an emerging link between mitochondria and ciliary dysfunction, and suggest that further understanding the enzymatic activity and substrates of XPNPEP3 will illuminate novel cystogenic pathways.

Conflict of interest: The authors have declared that no conflict of interest exists. Citation for this article: J Clin Invest. 2010;120(3):791-802. doi:10.1172/JCI40076.
Introduction

The autosomal recessive kidney disease nephronophthisis (NPHP) is the most frequent genetic cause of end-stage kidney disease in 


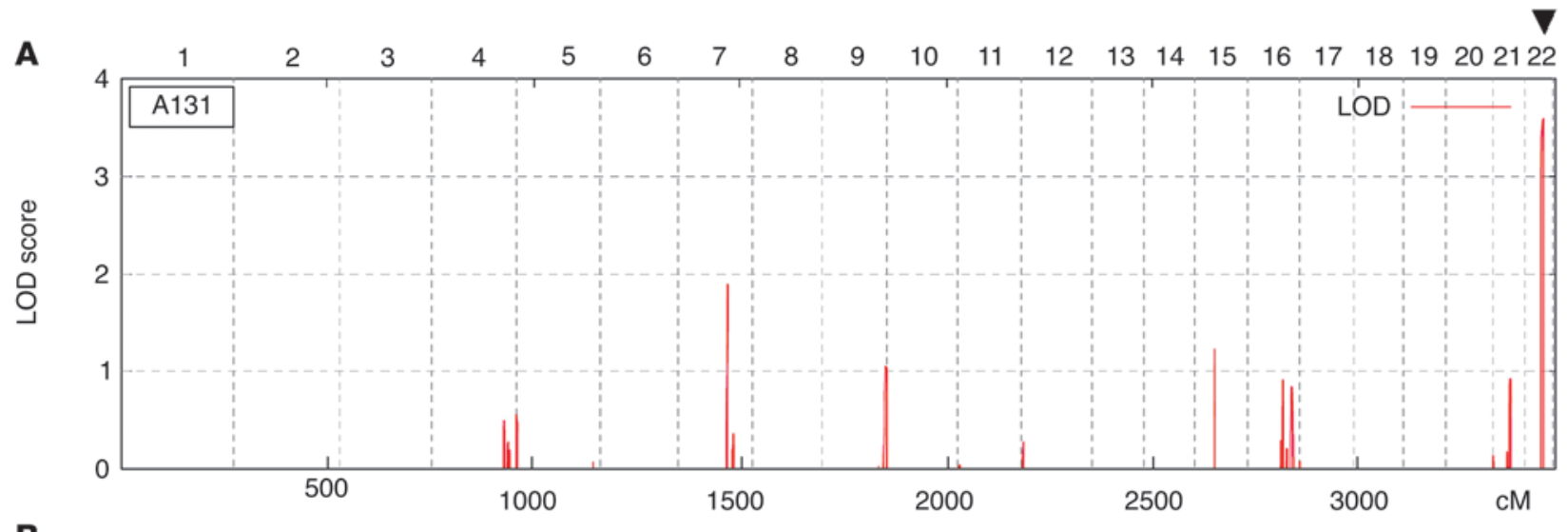

B
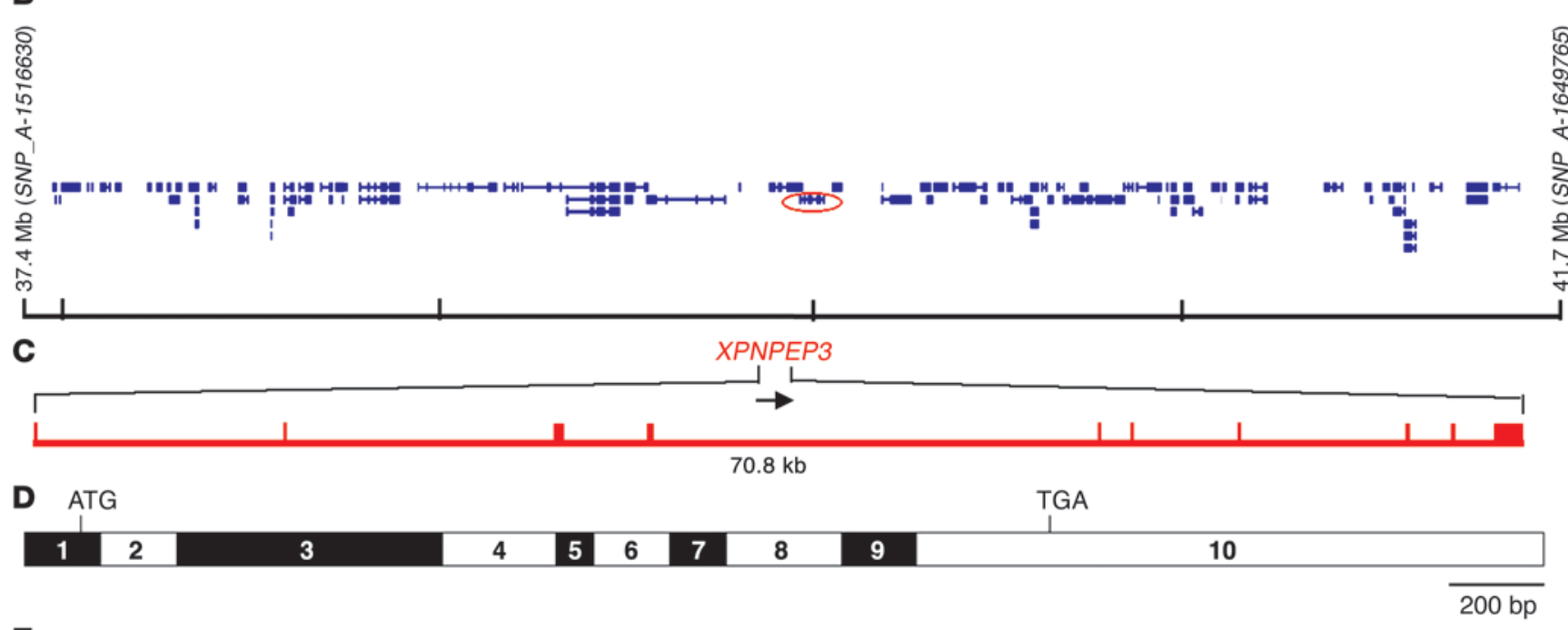

E

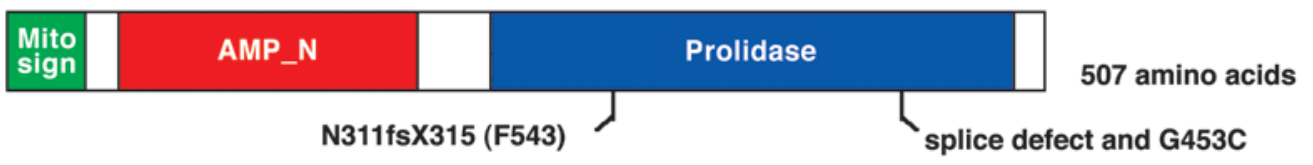

$\mathbf{F}$

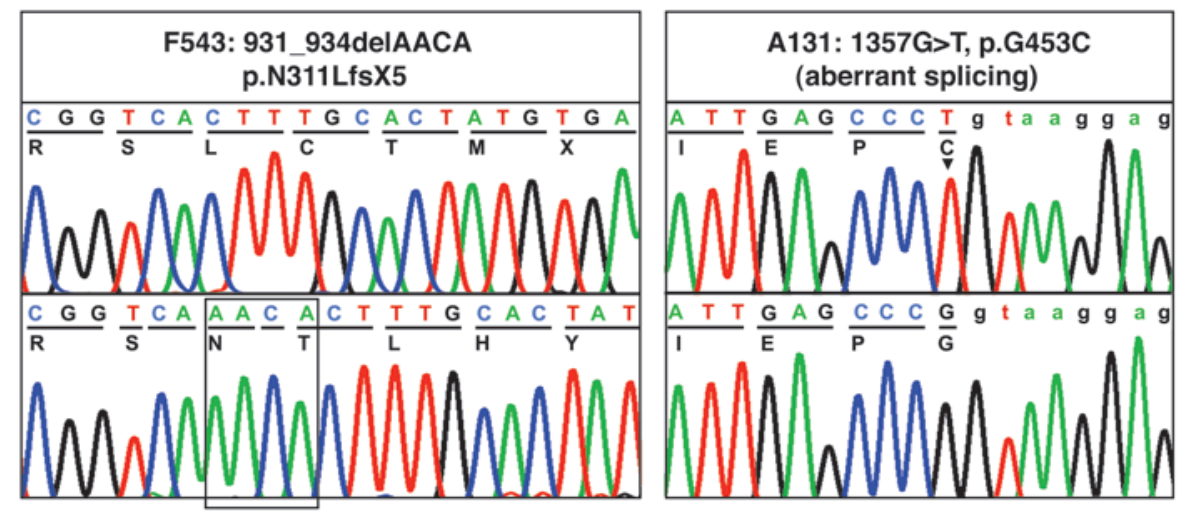




\section{Figure 1}

Positional cloning of the XPNPEP3 gene, as mutated in NPHPL1. (A) Parametric multipoint LOD score profile across the human genome of consanguineous kindred A131. Human chromosomes (numbered on top) are concatenated from pter (left) to qter (right) on the $x$-axis. Genetic distance is given in cM. Note the presence of a significant maximum LOD score of 3.6 on human chromosome 22q13.2 (arrowhead), defining a new gene locus (NPHPL1) for an NPHP-like kidney disease. (B) In kindred A131, the NPHPL1 locus, which is homozygous by descent, is delimited by heterozygous markers SNP_A-1516630 and SNP_A-1649765 to a 4.3-Mb interval, which contains 101 positional candidate genes (per the UCSC sequence; http://genome.ucsc. edu/). Mutations were detected in XPNPEP3 (encircled red). (C) The $X P N P E P 3$ gene extends over $70.8 \mathrm{~kb}$ and contains 10 exons (vertical hatches). (D) Exon structure of human full-length XPNPEP3 cDNA (3,056 bp). Positions of start codon (ATG) at nt +1 and of stop codon (TGA) are indicated. Exon sizes, ranging from $63 \mathrm{bp}$ to $997 \mathrm{bp}$, are approximated. (E) Positions of the mitochondrial localization signal (Mito sign). The SMART program (http://smart.embl-heidelberg.de) predicts a putative $\mathrm{N}$-terminal aminopeptidase $\mathrm{P}$ domain (AMP_N; amino acid 67-213), and prolidase domain (amino acid 253-490), which are drawn in relation to the encoding exon positions in $\mathbf{D}$. (F) Two homozygous mutations of XPNPEP3 detected in families A131 and F543 with NPHPL1 (see Table 1).

the first 3 decades of life (1). The characteristic histological findings in NPHP are renal interstitial infiltration with fibrosis, tubular atrophy with basement membrane disruption, and cyst development at the corticomedullary renal border (2). A combination of positional cloning and functional candidate gene approaches have led to the identification of 10 causative NPHP genes (NPHP1NPHP9 and NPHP11) (3-13). The products of each gene localize to primary cilia, basal bodies, and centrosomes, supporting a theory that describes cystic kidney diseases as "ciliopathies" $(5,6,14$, 15). Ciliary and centrosomal functions also provide a pathogenic basis for the extrarenal manifestations that can be associated with NPHP, including, among others, retinal degeneration in SeniorLoken syndrome (MIM \#609254) (15\%), cerebellar vermis aplasia in Joubert syndrome (MIM *610142) (10\%-15\%), and liver fibrosis (MIM 609884) (5\%) (1).

Mechanistically, several hypotheses have been put forth to explain the mechanism of cystogenesis in the absence of functional cilia. A mechanosensory-based hypothesis suggested that abnormal flow sensing is causally associated with cyst formation (16), whereas more recent data have indicated that defects in the axis of cell division and polarization of renal epithelial cells might drive the cystic phenotype (17). Interestingly, both models implicate defective Wnt signaling. In the mechanosensory model, fluid flow has been shown to activate INVS/NPHP2, which has been suggested to serve as a molecular modulator between canonical/ $\beta$-catenin and noncanonical/planar cell polarity (noncanonical/PCP) signaling (18). At the same time, PCP plays an important role in establishing polarity in developing tissues. In an Hnf-1 $\beta$ cystogenic model, it was shown that the plane of cell division was perturbed and tracked with cyst formation (19). Importantly, the same was shown for at least 2 PCP effectors, Fat4 and seahorse, in mice and zebrafish, respectively (20, 21). Furthermore, both we and others have shown that suppression of ciliary and basal body proteins, including BBS1, BBS4, BBS6, RPGRIP1L, and KIF3A, results in disruption between the balance of $\beta$-catenin and PCP signaling, in both renal epithelial cells in vitro and also in vivo zebrafish models, manifested as defective convergent extension movements during gastrulation $(22,23)$.
Because mutations in the known NPHP genes are found in only $35 \%$ of NPHP families, and many questions remain regarding the molecular pathogenesis of NPHP, we sought to clone additional disease-causing genes using whole-genome homozygosity mapping. Here we report causative mutations in the gene encoding $\mathrm{X}$-prolyl aminopeptidase 3 (XPNPEP3) as what we believe to be a new cause of an NPHP-like disorder. We demonstrate that XPNPEP3 localizes to mitochondria in renal cells in vitro and to kidney tubules in a cell type-specific pattern. Although, XPNPEP3 was undetectable in primary cilia or centrosomes, biochemical studies demonstrate that several cystogenic proteins are likely XPNPEP3 substrates, including NPHP6/CEP290, in which loss of function causes a range of ciliopathies, including $\operatorname{NPHP}(9,23-27)$. Our data implicate for the what we believe to be first time a role for mitochondrial proteins in the development of ciliopathy-like phenotypes and offer a potential mechanism for the cystogenic effect of XPNPEP3 loss of function.

\section{Results}

Mutations in XPNPEP3 cause an NPHP-like kidney disease. We performed a whole-genome search for linkage in 116 consanguineous kindred with NPHP and NPHP-like phenotypes. Linkage analysis in a 5-generation consanguineous family from northern Finland (A131), with 3 members affected with an NPHP-like disorder, yielded a significant logarithm of odds (LOD) score of $\mathrm{LOD}_{\text {max }}=3.6$, defining a new locus (NPHP-like 1, NPHPL1) on chromosome 22q13.2 (Figure 1A), within a 4.3-Mb interval flanked by markers SNP_A-1516630 and SNP_A-1649765 (Figure 1B). The critical genetic region overlapped with a homozygous segment detected in a consanguineous kindred of Turkish descent (F543), having 2 members with an NPHP-like phenotype (Supplemental Figure 1; supplemental material available online with this article; doi:10.1172/JCI40076DS1). The NPHPL1 critical genetic interval contained 101 positional candidate genes (Figure 1B).

We performed mutation analysis by sequencing exons in 29 of the candidate genes, prioritizing proteins present in the Ciliary proteome database (http://ciliaproteome.org/; ref. 28). Among them, we identified novel, likely pathogenic variants in only one gene, XPNPEP3 (Figure 1, B-F). Kindred A131 harbored a homozygous splice-site mutation $(1357 \mathrm{G}>\mathrm{T})$ in an $80 \%$ conserved exonic position of the splice donor consensus (Figure 1, B-F). RT-PCR from lymphoblastoid cells showed that this mutation disrupts correct splicing by activating a cryptic splice site and introducing a frame shift (Supplemental Figure 2). The same mutation changed an amino acid residue (G453C) that is conserved throughout evolution, including E. coli (protein ecAPP) (data not shown). In addition, the 2 affected individuals of kindred F543 had a homozygous 4-base deletion in exon 6 (c.931_934 delAACA), resulting in a premature stop codon (p.N311LfsX5) within the predicted prolidase domain, which is the catalytic domain (Figure 1, E and F). The mutation in family F543 segregated from both parents and was absent from 150 people of mixed European descent and 83 Turkish healthy control individuals. Likewise, the mutation of the northern Finnish family A131 segregated from both parents; it was absent from 118 Western European healthy control individuals (Table 1) and was present once heterozygously in 100 Finnish control individuals, where it occurred on a shared haplotype of more than $2 \mathrm{Mb}$, suggesting a founder effect.

We then examined 96 kindred with NPHP by direct sequencing of all exons and screened a multiethnic cohort of 376 additional kindred with NPHP using heteroduplex analysis (29) and 


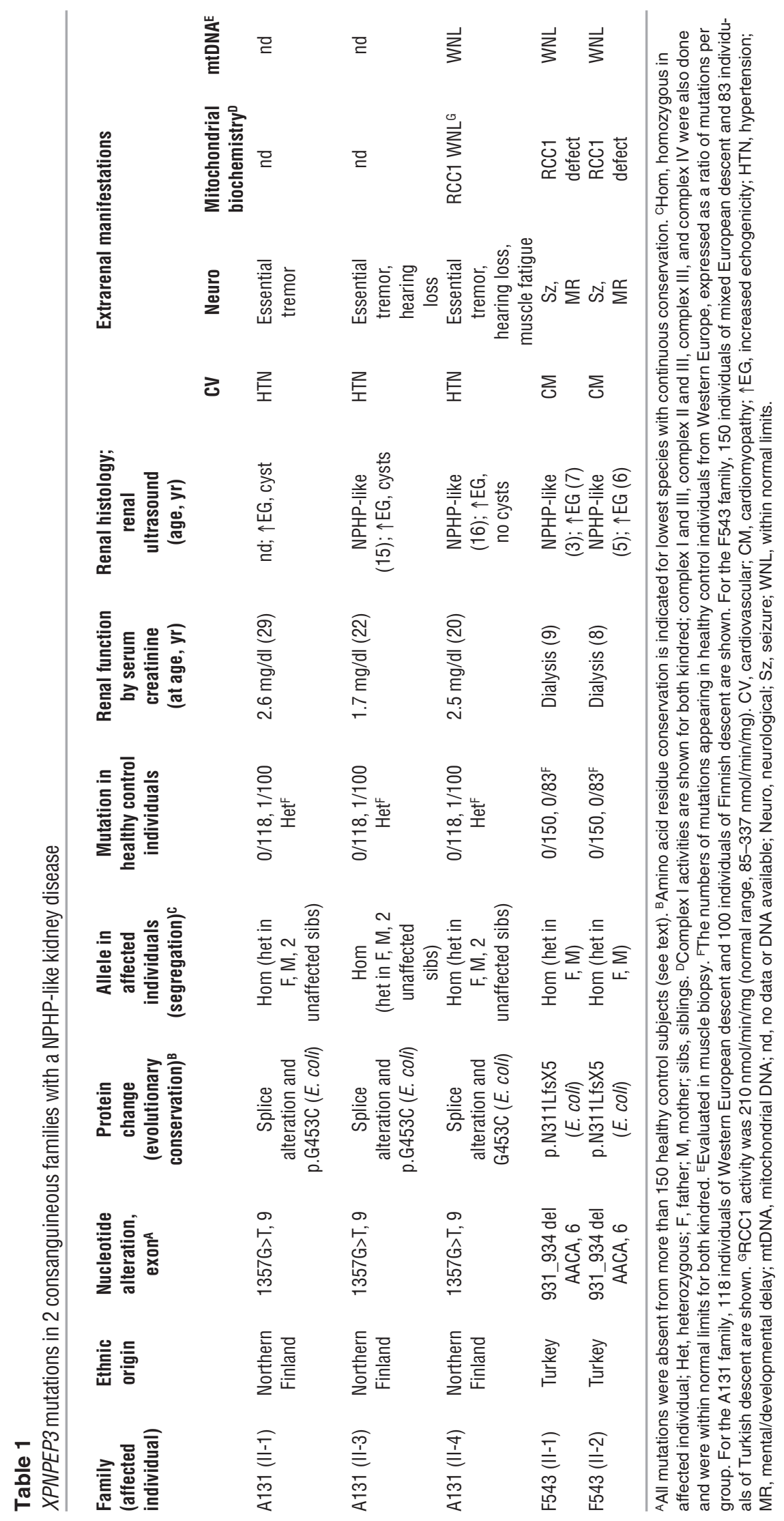

CELI digestion (30), followed by direct sequencing of all aberrant bands. We also examined 96 different kindred with Joubert syndrome and 95 different kindred with Senior-Loken syndrome using heteroduplex analysis (29), with subsequent direct sequencing of all aberrant bands. In addition, we analyzed 100 DNA samples from patients with isolated respiratory chain complex I (RCCI) deficiency, using high-resolution melting point analysis. We detected no patients with 2 mutations in any of these additional 643 families with NPHP-like phenotype and in 100 index cases with isolated RCCI deficiency. Ten novel heterozygous mutations of unknown pathogenicity were identified in 10 patients. We did not find any kindred with 2 recessive mutations in any of 823 families with an NPHP-like phenotype (Table 1).

Wethusidentified mutations in XPNPEP3 as a novel cause to our knowledge of an NPHP-like nephropathy (NPHPL1). XPNPEP3 is contained in the M24B subfamily of X-prolyl peptidases that comprises aminopeptidase $\mathrm{P}$ and the dipeptidase prolidase. XPNPEP3 has a predicted C-terminal catalytic domain and has greatest overall sequence identity (32\%) to E. coli aminopeptidase P. This high level of conservation, which includes the catalytic domain, suggests that XPNPEP3 is a functional aminopeptidase $\mathrm{P}$ and is 1 out of 3 $\mathrm{X}$-prolyl aminopeptidases known to exist in mammals (31) (Figure 1E). The E. coli ortholog and the human XPNPEP3 contain 2 manganese ions that are required for peptidase activity, sites that are also conserved in XPNPEP3 (31-33). Studies performed with the E. coli ortholog (34) indicate that XPNPEP3 recognizes N-terminal peptides that have proline in their second position as substrate and cleaves the peptide bond between the first and second amino acid residues, releasing the $\mathrm{N}$-terminal amino acid. In addition, MitoProt II analysis of XPNPEP3 detected an $\mathrm{N}$-terminal mitochondrial localization signal with a cleavage site after amino acid 53 , resulting in a $51-\mathrm{kDa}$ protein of likely mitochondrial function (Figure 1E).

Clinical and biochemical phenotype of individuals with NPHPL1. Clinical data of kindred A131 and F543 are presented in Table 1. Three affected individuals of kindred A131 had moderate renal insufficiency (glomerular filtration rate, $30 \%-40 \%$ of normal) at between 20 and 29 years of age (Table 1 ). Two individuals 


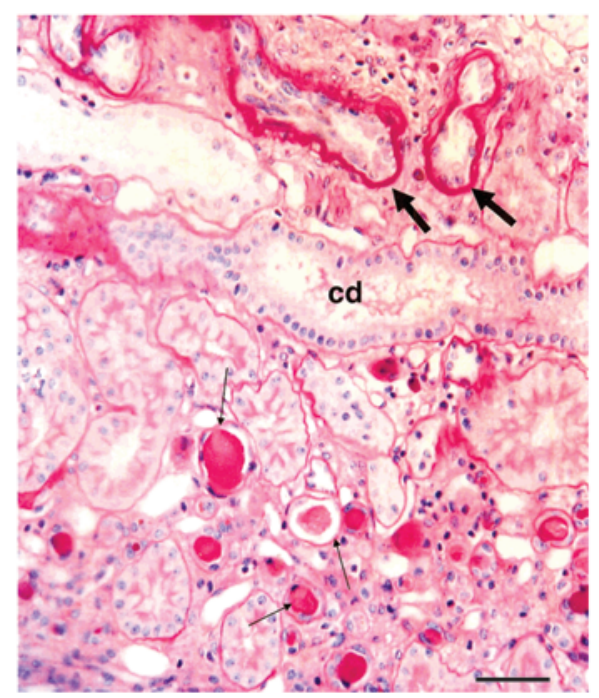

\section{Figure 2}

Renal histology of patient A131 II-4. Morphologic changes are characteristic for NPHP and include (a) thickening, splitting, and attenuation of tubular basement membranes in tubules with disorganized epithelium (thick arrows); (b) atrophic tubules that contain protein casts (thin arrows); (c) dilated collecting ducts (cd), which are lined by exceptionally tall epithelium; and (d) a mild degree of interstitial fibrosis. Most glomeruli appeared normal, but there were a few scattered obsolescent glomeruli (not shown). Scale bar: $30 \mu \mathrm{m}$.

of the northern Finnish family A131 had undergone renal biopsy, which yielded characteristic features of NPHP (Figure 2). Renal ultrasound revealed features characteristic of NPHP (Table 1), while extrarenal manifestations included essential tremor in all 3 affected individuals. Two affected individuals (A131 II-3 and A131 II-4) also had high frequency sensorineural hearing loss; A131 II-3 had arachnoid cysts on brain imaging, while A131 II-1 and A131 II-3 also had gout.

In the Turkish kindred (F543), the 2 affected individuals exhibited end-stage kidney disease by 8 and 9 years, respectively (Table 1). Renal ultrasound and renal histology demonstrated NPHP (data not shown). In addition, both affected individuals manifested a mitochondriopathy, with an isolated complex I deficiency and decreased NADH-CoQ oxidoreductase activity. This complex I deficiency was not found in fibroblast cultures from family A131, with the splice-site mutation (Supplemental Tables 2 and 3), but in family F543, with a homozygous frame-shift mutation, it was detected in a muscle biopsy of F543-I (Supplemental Tables 4 and 5) and in a fibroblast culture of F543-II (Supplemental Tables 6 and 7). Additionally, both affected individuals had seizures and hypertrophic dilated cardiomyopathy. F543 II-1 also demonstrated chronic pancreatitis with pancreatic cysts, while F543 II-2 had a hepatopathy.

A signal peptide traffics XPNPEP3 to mitochondria. To study the expression and subcellular localization of XPNPEP3, we characterized a rabbit polyclonal anti-human XPNPEP3 antibody (HPA000527, Sigma-Aldrich), hereafter called $\alpha$-XPNPEP3. Upon immunoblotting, $\alpha$-XPNPEP 3 specifically recognized transiently transfected V5-tagged human XPNPEP3 (XPNPEP3-V5) as well as endogenous XPNPEP3 in lysate from murine inner medullary collecting duct (IMCD3) cells (Supplemental Figure 3B). It also specifically detected overexpressed XPNPEP3-V5 in mitochondria, where it colocalized with the anti-V5 antibody (Supplemental Figure $3, \mathrm{~A}$ and $\mathrm{C}-\mathrm{E}$ ) as determined by immunofluorescent staining. Upon immunoblotting of cell lines from human, monkey, or dog, the $\alpha$-XPNPEP 3 cross-reacted with single bands at $51 \mathrm{kDa}$, representing the expected size for full-length XPNPEP3, following cleavage of the mitochondrial signal peptide (data not shown). Immunoblotting of multiple tissues from adult mouse detected single bands in cell lysates of kidney, heart, liver, skeletal muscle, brain, and testis (Supplemental Figure 4).

Because of the predicted targeting of XPNPEP3 to mitochondria, we fractionated whole kidney homogenates from mice into mitochondrial and cytosolic fractions. Immunoblotting with $\alpha$-XPNPEP3 yielded a single band in the mitochondrial fraction at approximately $51 \mathrm{kDa}$, consistent with the product predicted to result from cleavage of the mitochondrial signal peptide following mitochondrial import (Figure 3A). The cytosolic fraction showed a doublet at approximately $57 \mathrm{kDa}$, compatible with unprocessed XPNPEP3. Immunofluorescent microscopy in IMCD3 cells stably expressing human full-length XPNPEP3-GFP, which contains the mitochondrial leader sequence, were localized to mitochondria (Figure 3B). In contrast, in IMCD3 cells that stably express a cDNA, which lacks the mitochondrial leader sequence ( $\triangle \mathrm{N}$-XPNPEP3GFP), XPNPEP3 was found diffusely in the cytoplasm (Figure 3C). Expression of XPNPEP3 in mitochondria was also confirmed by transmission electron microscopy in ultrathin rat kidney sections, using immunogold-labeled $\alpha$-XPNPEP3 (Figure 3D).

No evidence for XPNPEP3 in primary cilia, basal bodies, and centrosomes in vitro. NPHP is considered a ciliopathy, because 10 different causative genes (NPHP1-NPHP9 and NPHP11) share the feature of subcellular function of their respective gene products at primary cilia, basal bodies, or centrosomes (1). We therefore examined XPNPEP3 for organellar targeting to the cilia/basal body/centrosome complex. In IMCD3 cells that stably express the human full-length XPNPEP3-GFP, which contains the mitochondrial leader sequence, XPNPEP3-GFP was not detected in primary cilia, basal bodies, or centrosomes (Figure 4). We conclude that loss of XPNPEP3 leads to an NPHP-like phenotype (NPHPL1), without any apparent ciliary localization, departing from the paradigm of all other genes mutated in NPHP.

XPNPEP3 exbibits cell type-specific expression in kidney. As the most prominent phenotype in individuals with suppressed XPNPEP3 function was manifested in the kidney, we performed immunofluorescent microscopy of rat kidney sections to study cell type-specific expression. We found XPNPEP3 to be expressed specifically in distal convoluted tubule and cortical collecting duct cells (Supplemental Figure 5). Of note, XPNPEP3 expression was detected not in principal cells but in intercalated cells, which are known to lack primary cilia (Supplemental Figure 5).

xpnpep3 suppression phenocopies ciliary and basal body zebrafish morphants. Depletion of several cystogenic proteins, including NPHP2, CEP290/NPHP6, and numerous BBS proteins, have been shown to perturb convergence and extension movements in zebrafish $(18,22$, $25,35)$, a noncanonical Wnt phenotype, which in the mammalian nephron manifests as failure of orientation of the axis of cell division and has been causally linked to tubular dilatation (20). We therefore wondered whether suppression of xpnpep 3 might phenocopy this defect or whether loss of this gene/protein might affect hitherto unknown pathways. We therefore designed a translationblocking morpholino (MO) against the sole Danio rerio ortholog (by reciprocal BLAST), a transcript that is expressed as early as 12 hours 

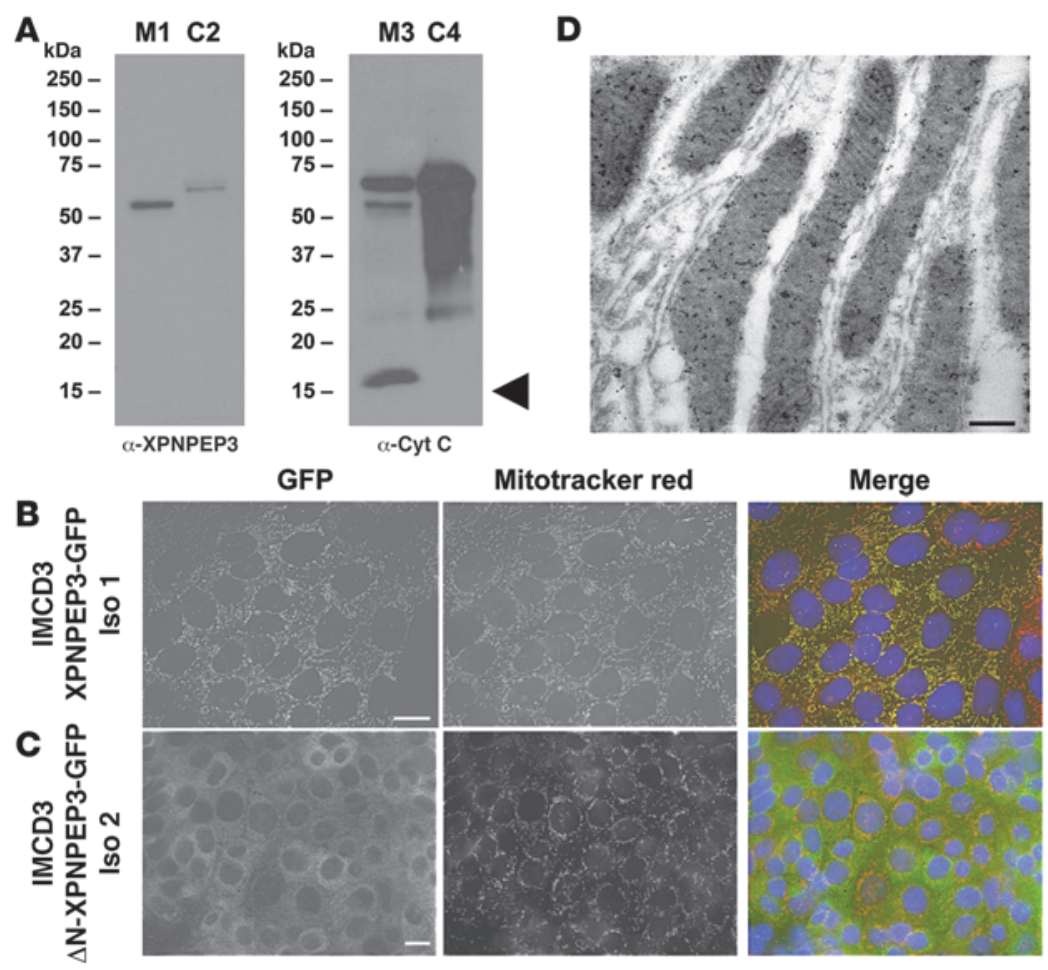

Figure 3

Human XPNPEP3 is targeted to mitochondria by an amino-terminal mitochondrial signal sequence. (A) Whole kidney homogenates from mice were fractionated into mitochondrial fractions (lanes M1 and M3) and cytosolic fractions (lanes C2 and C4). Immunoblotting with $\alpha$-XPNPEP3 yielded a single band in the mitochondrial fraction at approximately $51 \mathrm{kDa}$ (lane M1), consistent with the product of cleavage of the mitochondrial signal peptide (after amino acid 53) predicted to occur after mitochondrial import. A doublet was seen in the cytosolic fraction (lane C2) at approximately $57 \mathrm{kDa}$, which is compatible with the full-length isoform 1 of XPNPEP3. Reblotting with a monoclonal antibody against the mitochondrial protein cytochrome $c$ (Cyt C) demonstrates that cytochrome $c$ is enriched in the mitochondrial fraction (lane M3), yielding a band at approximately $15 \mathrm{kDa}$ (arrowhead), which was absent from the cytosolic fraction (lane C4). (B) Immunofluorescent microscopy was performed on IMCD3 cells that stably express the human full-length XPNPEP3-GFP (in $90 \%$ of cells) (left panel), which contains the mitochondrial leader sequence. Upon costaining with the mitochondrial marker, Mitotracker Red, there was colocalization (right panel) in mitochondria. Scale bar: $10 \mu \mathrm{m}$. Iso 1, isoform 1. (C) By contrast, in IMCD3 cells that stably express an amino-terminal deletion construct of XPNPEP3-GFP, lacking the 53-amino acid N-terminal mitochondrial signal sequence ( $\triangle N$-XPNPEP3-GFP) (left panel), XPNPEP3 expression occurred diffusely in the cytoplasm rather than in mitochondria (right panel). Mitochondria were again labeled with the marker Mitotracker Red. Scale bar: $10 \mu \mathrm{m}$. (D) To detect presence of endogenous Xpnpep3 in mitochondria of distal tubular segments in rat kidney, $\alpha$-XPNPEP3 was labeled with 10-nm gold particles. Endogenous Xpnpep3 labeling was detected in mitochondria of distal tubular segments. Scale bar: $1 \mu \mathrm{m}$.

after fertilization, as determined by RT-PCR (data not shown). At the mid-somite stage, embryos injected with xpпpep3 $\mathrm{MO}$ ( $n=50-100$ embryos/injection; scored blind) displayed quantifiable gastrulation defects reminiscent of ciliary gene morphants $(22,25$, 35 ); these included a shortened body axis, small anterior structures, broadening and kinking of the notochord, and elongated somites (Supplemental Figure 6). These phenotypes were unlikely to be caused by nonspecific action of the MO: not only did we observe an increase in affected embryos correlated with dose, but rescue with capped full-length human XPNPEP3 mRNA resulted in significant amelioration of the phenotype $\left(\chi^{2}=34.45, P<0.0001\right)$. These phenotypes are likely independent of the XPNPEP3 mitochondrial activity: coinjection of $x p$ npep $3 \mathrm{MO}$ with a human rescue construct devoid of the mitochondrial localization signal ( $\triangle \mathrm{N}-X P N P E P 3)$ rescued the phenotype in a manner indistinguishable from that of the WT human construct $\left(\chi^{2}=3.47, P=0.17\right)$. Therefore, targeting of XPNPEP3 to the mitochondrion is not required to rescue the convergence and extension phenotypes, suggesting that this protein might also have mitochondrial-independent activity.
Cleavage of known cystic disease proteins by XPNPEP3. Our data led us to hypothesize that, in addition to its mitochondrial functions (which potentially explain the RCCI defects observed in family F543), XPNPEP3 might affect the function of ciliary proteins biochemically, most likely through its $\mathrm{N}$-terminal proline cleavage activity. To identify such candidate substrates, we parsed the ciliary proteome (28) for proteins with a proline in the second position (after $\mathrm{N}$-terminal methionine cleavage). To reduce incidence of false-positives, we used a stringent version of the proteome that was restricted to reciprocal orthologs (minimal $E$ value of $10^{-30}$ ) identified in at least 2 independent ciliary studies, thereby parsing 426 likely ciliary proteins for substrate candidacy. We identified 51 proteins with sequences that fulfilled our search criteria (Supplemental Table 8). Interestingly, 3 candidate substrates (centrosomal protein $290 \mathrm{kDa} / \mathrm{NPHP} 6$ [CEP290/NPHP6], Alstrom syndrome 1 [ALMS1], and leucine rich repeat containing 50 [LRRC50]) are known to cause cystic renal disease $(9,36,37)$.

To verify our computational predictions, we next sought to test whether XPNPEP3 can cleave these 3 candidate substrates. Expression of recombinant human XPNPEP3 in bacteria was not pos- 
A

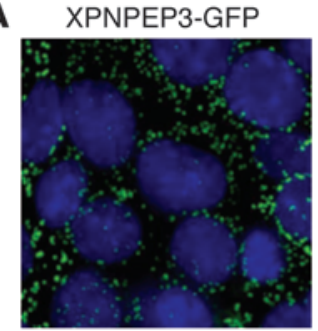

B

XPNPEP3-GFP

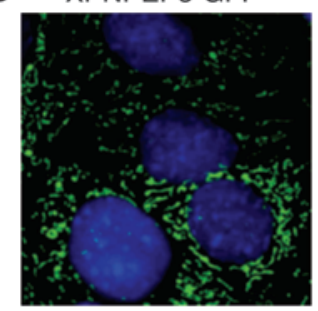

C

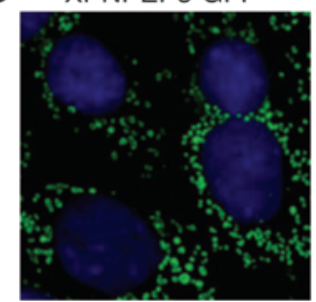

Ac- $\alpha$-Tubulin, Alexa 594

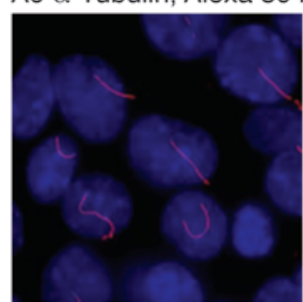

$\gamma$-Tubulin, Alexa 594

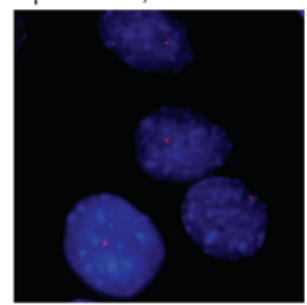

Pericentrin, Alexa 594

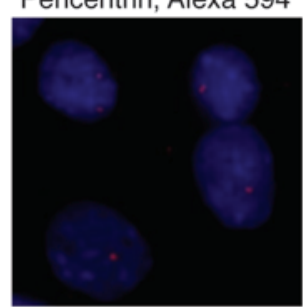

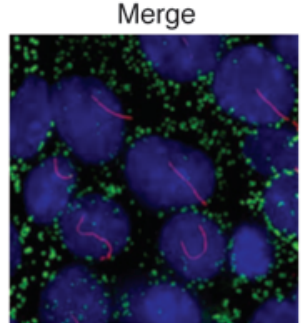

Merge

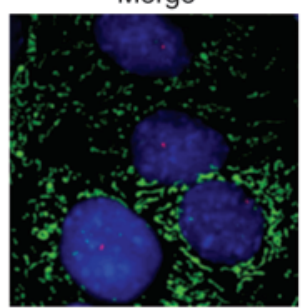

Merge

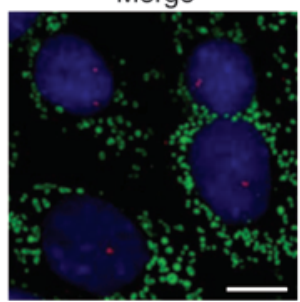

Figure 4

Full-length human XPNPEP3-GFP transiently expressed in IMCD3 cells localizes to mitochondria and not to the cilium/basal body/centrosome complex in IMCD3 cells. Immunofluorescent microscopy was performed in IMCD3 cells that stably express human full-length XPNPEP3-GFP, which contains the mitochondrial signal sequence, demonstrating expression of XPNPEP3 in mitochondria. XPNPEP3-GFP was not detected in primary cilia $(\mathbf{A})$, basal bodies (B), or centrosomes (C), when counterstaining with acetylated $\alpha$-tubulin (Ac- $\alpha$-tublin) (A), $\gamma$-tubulin (B), or pericentrin (C), respectively (middle and right panels). Scale bar: $10 \mu \mathrm{m}$.

sible due to the protein being insoluble under numerous experimental conditions. We therefore focused on the E. coli ortholog of XPNPEP3, ecAPP, which we were able to express and purify to more than $99 \%$ homogeneity as detected by Coomassie staining and Western blot (Figure 5A). Three 9-amino acid peptides identical to the N-termini of each of CEP290/NPHP6, ALMS1, and LRRC50 (without the start methionine that is cleaved after translation) were synthesized (Figure 5B) and incubated with purified ecAPP, followed by mass spectrometry. We found that CEP290/NPHP6, ALMS1, and LRRC50 are each cleaved efficiently by ecAPP (Figure 5C). By contrast, peptides containing residues other than proline in the second position could not be processed by ecAPP (data not shown). Importantly, the cleavage activity of the enzyme is not promiscuous, since we found that dynein, another ciliary protein that also contains a proline in the second residue but is not involved in cystic kidney diseases, is poorly digested by ecAPP (Figure 5C). These data suggested that XPNPEP3 cleavage of other cystogenic proteins might be relevant to their biological function(s).

Requirement of XPNPEP3 N-terminal cleavage for early gastrulation in zebrafish. To probe the importance of the likely XPNPEP3 cleavage sites in vivo, we asked whether cleavage resistance was deleterious

to protein function. Focusing on LRRC50 (each of CEP290/ NPHP6 and ALMS1 encode mRNA more than $7 \mathrm{~kb}$, rendering them difficult to transcribe in vitro at sufficient purity), we investigated whether a cleavage-resistant human capped mRNA can rescue the gastrulation phenotypes caused by MO-driven suppression of endogenous lrrc50. Injection of $4.5 \mathrm{ng}$ of a translation-blocking $\mathrm{MO}$ induced early gastrulation phenotypes that include all the hallmarks of cilia-related convergent extension defects and which could be rescued by coinjection of $150 \mathrm{pg}$ of WT human capped LRRC50 mRNA (Figure 6). However, coinjection of MO with mRNA encoding an N-terminal Pro-Val point mutation, followed by blind scoring of embryos, showed complete failure of rescue (WT vs. Pro-Val, $\chi^{2}=39.20, P<0.0001 ; n=80-110$ embryos $/$ injection). This experiment could not exclude the possibility that the observed loss of function could be due to the presence of the $\mathrm{Val}$ at this position. However, repetition of this assay with Asp or Arg yielded similar data (Figure 6). Still, the possibility remained that the observed failure to rescue might still be driven by the loss of the proline for reasons other than cleavage. To assess this possibility, we searched the zebrafish genome for other N-terminal aminopeptidases and identified an alanyl aminopeptidase (ZFIN ID, ZDB-Gene-030131-1253), which predicts that Ala at the position of the $\mathrm{N}$-terminal proline should render lrrc50 subject to cleavage. Consistent with the notion that it is the cleavage that functionalizes $\operatorname{lrrc50}$, injection of MO with an Ala-encoding human mRNA rescued the phenotypes in a manner indistinguishable from WT (Figure 6; $\chi^{2}=0.26, P<0.87$ ).

\section{Discussion}

Using a whole-genome homozygosity mapping approach, we hereby identify recessive mutations in XPNPEP3 as a new cause of an NPHP-like disease. The phenotypic severity and organ involvement vary widely between the 2 different families with XPNPEP 3 mutations, whereas the phenotypic spectrum is concordant for affected individuals within the same family (Table 1). Specifically, 3 affected individuals of family A131 exhibited only a mild form of an NPHP-like kidney disease (Figure 2), with residual kidney function beyond the age of 20 years, at which time virtually all patients with "classic" NPHP would have reached ESRD (1). Extrarenal manifestations were limited to mild neurologic involvement with sensorineural hearing loss and essential tremor (Table 1). In contrast, the 2 affected siblings of F543 exhibited early-onset renal failure, leading to renal replacement therapy at 8 and 9 years of age, with extrarenal manifestations of mental retardation, seizures, and cardiomyopathy. The observed phenotypic variability might be due to different degrees of loss of function for the 2 different homozygous XPNPEP 3 alleles. Whereas in family A131, residual correct splicing of the $80 \%$ conserved splice consensus may produce some WT splice product and thereby residual function (Supplemental Figure 2), the frameshift mutation of F543 will result in complete truncation of the C-terminal third of XPNPEP 3 and likely renders the mutant message substrate for nonsense-mediated decay. This notion is also supported by the finding that there was a measurable reduction in RCCI function in muscle biopsy samples of F543-I and fibroblasts of F543-II but not those of family A131. 


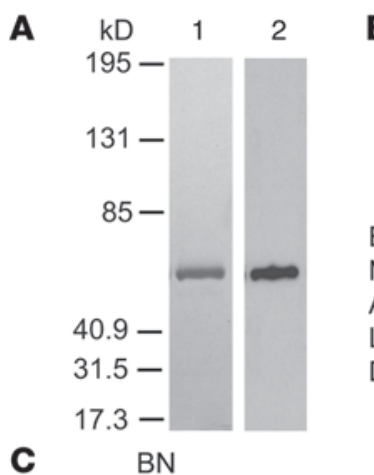

Before digestion
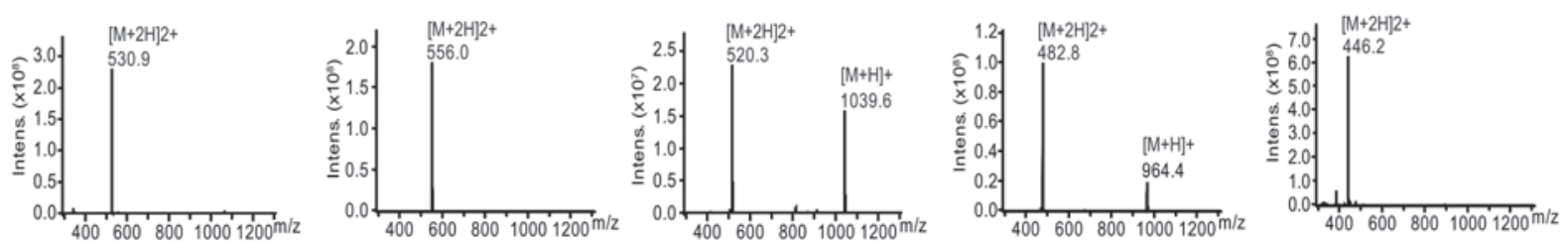

After digestion
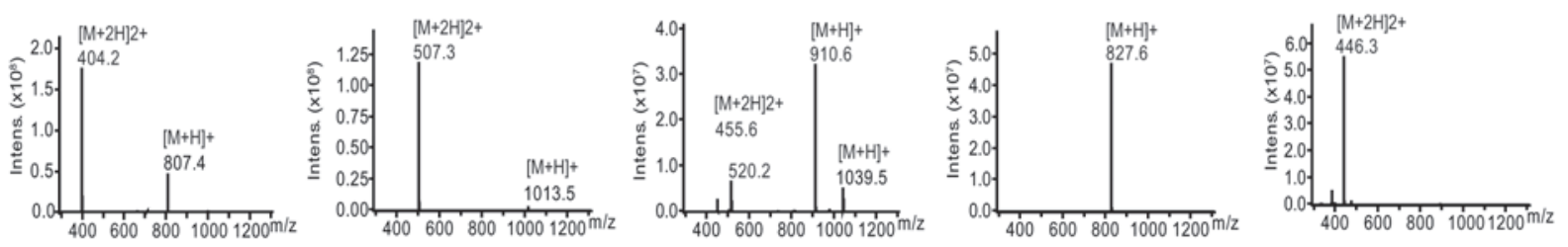

\section{Figure 5}

Peptide cleavage by the XPNPEP3 ortholog, ecAPP. (A) Expression and purification of ecAPP. Coomassie staining of purified protein (lane 1) and immunoblot with anti-His tag antibody (lane 2) are shown. (B) Peptide substrates designed for enzymatic assay. Arrows indicate the cleavage site(s). Proline residue recognized by ecAPP is highlighted in red. Molecular weights are listed in Da. BN, bradykinin; N6, CEP290/NPHP6; AL, ALMS1; LR, LRRC50; DN, dynein. (C) Peptide cleavage detected by electrospray ionization-liquid chromatography-mass spectrometry. Mass spectrometry of peptides before and after digestion is shown. $\mathrm{M}+n \mathrm{H}$ indicates the addition of $n$ protons to the mass after ionization. intens, intensity.

Alternatively, modifiers in either family might potentiate or protect the effect of the respective primary mutations, as has been suggested for other ciliopathies $(23,38)$.

NPHP is considered a ciliopathy because 10 different causative genes (NPHP1-NPHP9 and NPHP11) share the feature of subcellular expression of their respective gene products at primary cilia, basal bodies, or centrosomes (1). The absence of XPNPEP3 in cilia or basal body from in vitro cell culture could be due to the limitation of antibody affinity, or epitope internalization within cilium, or requirement of further posttranslational procession of XPNPEP3 before localizing to that organelle. It is also possible that trafficking of XPNPEP3 to the cilium or basal body requires some specific physiological stimulus, as shown for Smo (39).

Nevertheless, our data suggest a mitochondria-independent, yet cilia-related function, for XPNPEP3. Suppression of $x p n p e p 3$ in zebrafish phenocopies the developmental phenotypes induced by the suppression of numerous ciliary and basal body-encoding genes $(22,23,25)$. Importantly, these phenotypes can be rescued by the хрпрер 3 isoform devoid of mitochondrial targeting sequence, which in mammalian cells leads to cytoplasmic diffusion of the protein. We propose the ciliary phenotypes unmasked by loss of XPNPEP3 might arise from the loss of XPNPEP3-dependent processing of ciliary proteins. This idea is reinforced by the observed requirement to cleave the $\mathrm{N}$-terminal proline of 3 cystogenic pro- teins, most likely executed by XPNPEP3, and by the demonstration that, at least for LRRC50, N-terminal proline cleavage is necessary for correct gastrulation movements. Given the observed localization of XPNPEP3 in mammalian renal cells, we speculate that such processing might occur outside the cilium, especially since there is mounting evidence for the requirement of ciliary proteins in preciliary functions that include vesicular transport to the apical membrane/transition zone (41) and for the assembly of some intraflagellar transport particles in the cytoplasm (42). Although, in the present study CEP290/NPHP6, ALMS1, and LRRC50 (loss of each of which can induce renal cysts), have been identified as likely targets of XPNPEP3, we do not know whether it is the dysfunction of these molecules that drives NPHP in the 2 families with XPNPEP3 mutations. It will be important to assay the maturation of these 3 proteins in patient cells and to examine the possible anatomical and signaling defects of primary renal cilia in the absence of XPNPEP3.

\section{Methods}

Study subjects. We obtained blood samples, clinical data, and pedigree information after obtaining informed consent from patients with NPHP and/or their parents. Approval for these studies was obtained from the University of Michigan Institutional Review Board. The diagnosis of NPHP, as defined by the Online Mendelian Inheritance in Man database, was based 
A

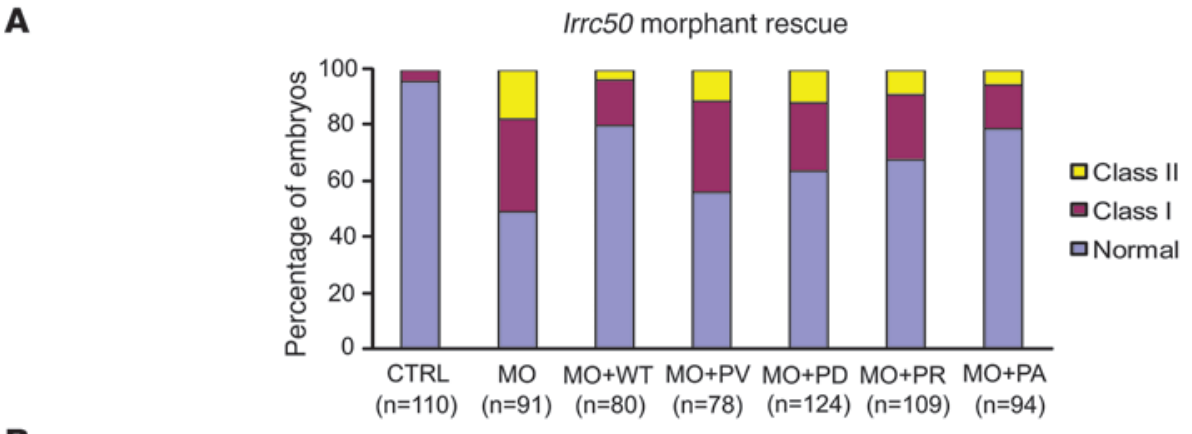

B
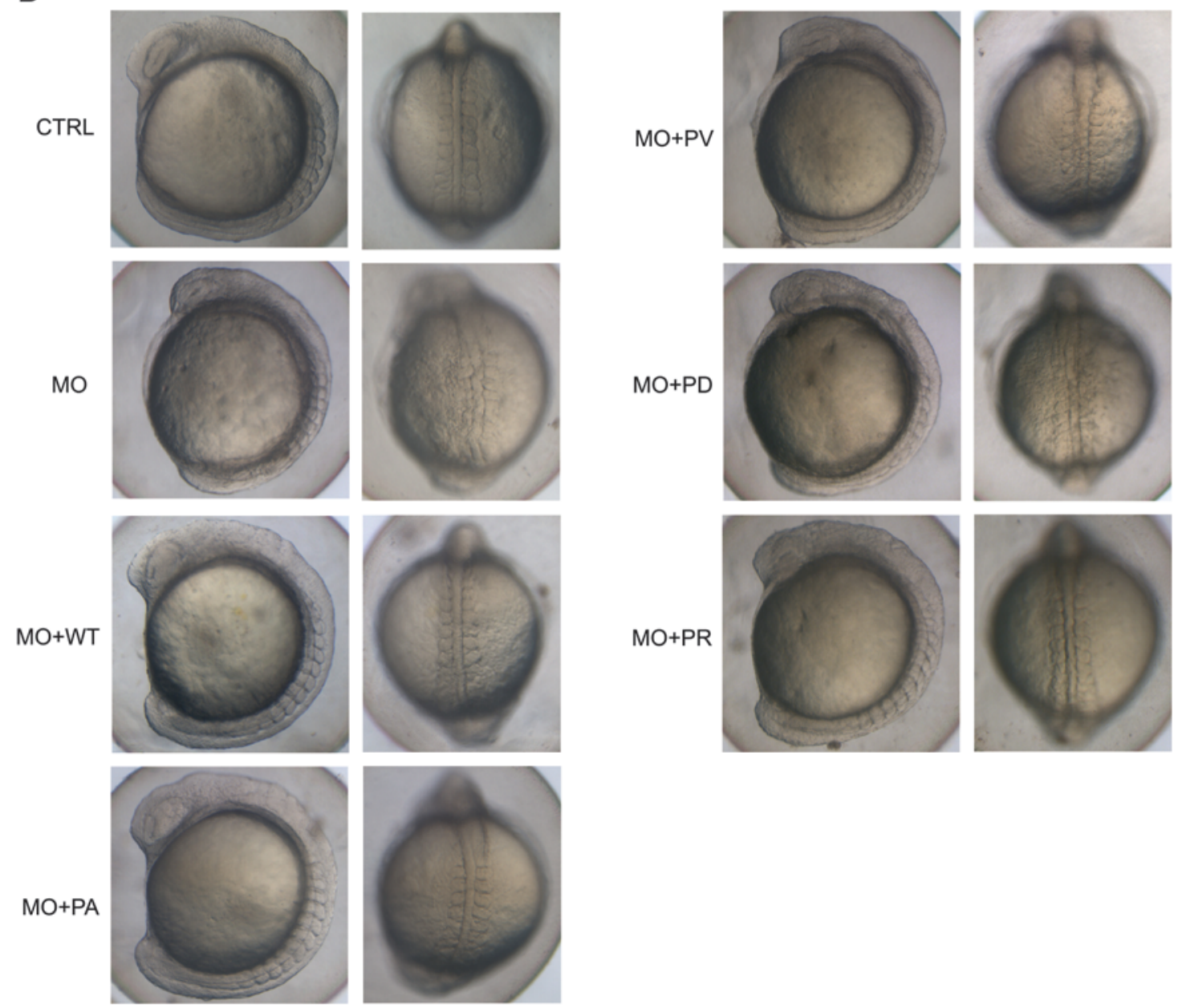

\section{Figure 6}

$\mathrm{N}$-terminal cleavage of LRRC50 is required to rescue Irrc50 morphant phenotypes in zebrafish. (A) Quantitative representation of the effect of Irrc50 MO on gastrulation development and rescue efficiency of different human Irrc50 RNA isoforms. The developmental phenotype of embryos was scored as Class I-II as described previously (22). Normal, indistinguishable from WT; Class I, mildly affected with a shortened body axis, small anterior structures, mild somite defects; Class II, severely affected with a short body axis, poorly defined head and eyes, broadening and kinking of the notochord, broad, thin somites, and tail extension defects. (B) Representative examples of embryos showing the gastrulation defect caused by Irrc50 MO, which could be rescued by WT and proline-to-alanine human LRRC50 but not other mutants. PA, proline to alanine; PV, proline to valine; PD, proline to aspartic acid; PR, proline to arginine.

on the clinical course and renal ultrasound or renal biopsy that were compatible with the diagnosis of NPHP, as judged by a pediatric nephrologist. Patients were selected for additional mutation analysis using the criteria that they had an isolated RCCI deficiency and a residual RCCI activity beneath the lowest control value from $5 \%$ to $90 \%$, with a median of $51 \%$.
Renal involvement was recorded for 11 out of 100 of those patients. Ten patients had renal tubular acidosis and one had renal insufficiency.

SNP genotyping and sequencing. Whole-genome search for linkage was performed in 116 consanguineous families with NPHP using an Affymetrix SNP array $(50 \mathrm{~K})$. Data were evaluated with nonparametric LOD 
scores across the entire genome to identify regions of homozygosity by descent as reported previously (43). Genomic segments of homozygosity were confirmed by high-resolution haplotype analysis within those regions using microsatellite markers. Additional SNPs were typed by direct sequencing. The GeneHunter program was used to calculate multipoint LOD scores, assuming recessive inheritance with complete penetrance, a disease allele frequency of 0.001 , and allele frequencies for northern European descent (Affymetrix).

Mutational analysis of candidate genes. Exon PCR of candidate genes was performed using genomic DNA from affected individuals. Exon-flanking primers were designed using the University of California, Santa Cruz sequence (http://genome.ucsc.edu/) and Primer3 software (http://ihg2. helmholtz-muenchen.de/ihg/ExonPrimer.html). PCR products were purified (Marligen Biosciences) prior to direct sequencing (Genetic Analyzer 3700, Applied Biosystems). Sequence data were analyzed using the software Mutation Surveyor (SoftGenetics) and Sequencher (Gene Codes). One hundred ninety healthy control individuals were screened as negative controls for each mutation identified.

High-resolution melting analysis was used for mutation scanning technology to analyze the coding region of XPNPEP3 in 100 patients with isolated RCCI deficiency. XPNPEP3 exons were PCR amplified from 5 ng genomic DNA, with a final denaturation step at $94^{\circ} \mathrm{C}$ for 1 minute (0.25 units Thermo-Start Taq DNA polymerase [Abgene], $1 \times$ LCGreen Plus [BIOKE], $0.25 \mu \mathrm{M}$ of each primer; Supplemental Tables 1-7). High-resolution melting analysis was performed on a LightScanner instrument (Idaho Technology). In the presence of the saturating double-stranded DNA-binding dye, amplicons were denatured, starting at $77^{\circ} \mathrm{C}$, while fluorescence intensities were recorded continuously. Melting curves were analyzed by LightScanner software (Idaho Technology), with normalized, temperature-shifted curves displayed as difference plots $(-d F / d T)$. Detected samples with altered melting curves, compared with the average of multiple WT results, were directly sequenced with the BigDye Cycle sequencing kit (Applied Biosystems) (44).

Antibodies and protein expression studies. The rabbit polyclonal $\alpha$-XPNPEP3 was from Sigma-Aldrich (HPA000527). Secondary antibodies to rabbit, mouse, and goat IgG were conjugated with either Alexa Fluor 488 or 594 (Molecular Probes). Antibodies for $\beta$-actin and acetylated $\alpha$-tubulin and $\gamma$-tubulin were from Sigma-Aldrich; V5 was from Invitrogen; and pericentrin was from Novus. The aquaporin-2 antibody was a goat polyclonal antibody from Santa Cruz Biotechnology Inc. (sc-9882). The cytochrome C oxidase antibody was a mouse monoclonal antibody from Santa Cruz Biotechnology Inc. (sc-658348).

Cloning. Full-length XPNPEP3 and $\triangle \mathrm{N}$-XPNPEP3 (amino acid 79-507) were PCR amplified from PENTR-XPNPEP3 (NM_022098.2, clone IOH5999; Invitrogen), with the termination codon removed and inserted into the pDONR221 vector, using BP Clonase II (Invitrogen), and subsequently transferred into pEF5/FRT/V5-DEST (Invitrogen) and pG-LAP5 (C-terminal GFP-Lap tag) (45) or pCS2+ vectors, using LR Clonase II (Invitrogen). Human LRRC50 was cloned into the $\mathrm{PCS} 2+$ vector; vectors encoding mutated LRRC50, with the third proline residue replaced by alanine, valine, aspartic acid, or arginine, were generated by site-directed mutagenesis. The fidelity of all constructs was confirmed by sequence analysis. MitoProt II was used for prediction of mitochondrial localization signal (MitoProt, http://ihg2.helmholtz-muenchen.de/ihg/mitoprot.html).

Immunoblotting. Immunoblotting was performed as described previously (9). For mitochondrial fractionation studies, whole kidneys from 6-month-old male 12956 mice were isolated, homogenized, and fractionated with differential centrifugation into mitochondrial and cytosolic fractions. Then, $50 \mu \mathrm{g}$ total protein from each fraction was loaded and separated on a denaturing $4 \%-12 \%$ SDS-PAGE gradient gel and trans- ferred to PVDF membrane. Total protein was visualized using Ponceau S staining. Membranes were then immunoblotted with $\alpha$-XPNPEP3 overnight at $4^{\circ} \mathrm{C}$ at a titer of 1:500.

Immunoelectron microscopy. Kidney cortex and medulla from 4-monthold Sprague-Dawley rats were used for immunocytochemical analysis. Tissues were fixed lightly for 5 minutes at $4{ }^{\circ} \mathrm{C}$ with $3 \%$ formaldehyde and $20 \mathrm{mM}$ ethylacetimidate in HEPES-buffered saline (30 $\mathrm{mM}$ HEPES, $151 \mathrm{mM} \mathrm{NaCl}, 4.7 \mathrm{mM} \mathrm{KCl}, 1.2 \mathrm{mM} \mathrm{MgCl}_{2}, 7.8 \mathrm{mM}$ glucose, $\left.\mathrm{pH} 7.3\right)$ at $4^{\circ} \mathrm{C}$. These samples were washed, further fixed in $3 \%$ formaldehyde containing $0.25 \%$ glutaraldehyde in HEPES-buffered saline at $4^{\circ} \mathrm{C}$ for 45 minutes, then dehydrated in ethanol and embedded in LR White Resin (Polysciences Inc.). Thin sections were blocked with PBS containing $1 \% \mathrm{w} / \mathrm{v}$ BSA and $0.01 \% \mathrm{v} / \mathrm{v}$ Tween 20 (PBT). Grids were then incubated with $\alpha$-XPNPEP3 (HAP000527, Sigma-Aldrich) at 1:10 and 1:25 dilution in PBT for 12 hours at $4{ }^{\circ} \mathrm{C}$. Negative controls included normal rabbit serum and PBT replaced as the primary antibody. After washing, grids were incubated for 1 hour in 10-nm gold-conjugated goat anti-rabbit IgG (Amersham Life Sciences) diluted 1:30 in PBT, rinsed with PBS, and fixed with glutaraldehyde to stabilize the gold particles. Samples were stained with uranyl acetate and lead citrate, then examined in a Zeiss CEM902 electron microscope.

Tissue culture. XPNPEP3-V5- and XPNPEP3-LAP-expressing cell lines were generated by cotransfecting IMCD3 Flp-In cells (Invitrogen) with pOG44 and PDEST EF/FRT XPNPEP3-V5 or PG-LAP5-XPNPEP3, according to the manufacturer's instructions. Stable cells were generated by selection with hygromycin $(800 \mu \mathrm{g} / \mathrm{ml})$.

Measurements of mitochondrial respiratory complex function were performed in muscle biopsy samples from family F543 as described previously $(46,47)$. In individual A131 II-4, mitochondria were isolated from a muscle biopsy sample using the method of Rasmussen et al. (48), and the respiratory chain complex activities were measured according to the method of Rustin et al. (49). An Aminco DW-2 Dual-Wavelength Spectrophotometer was used to record the enzyme kinetics.

Immunofluorescence studies in IMCD 3 cells. Cells were grown on 24-well glass bottom plates, stained with Mitotracker Red CMTMRos (250 nM) (Invitrogen) for 30 minutes at $37^{\circ} \mathrm{C}$ in $5 \% \mathrm{CO}_{2}$, and fixed with $4 \%$ paraformaldehyde. For indirect immunofluorescence studies, fixed cells were permeabilized with $0.1 \%$ Triton $/ 1 \%$ BSA/PBS, $\mathrm{pH} 7.4$, and stained with primary antibodies for 1 hour at $25^{\circ} \mathrm{C}$, followed by secondary antibody immunolabeling. Nuclei were counterstained with Hoechst (Invitrogen). Epifluorescence microscopy images were acquired using a Zeiss AxioObserver.Z1, with a $63 \times 1.4$ numerical aperture oil objective, and a Hamamatsu ORCA-ER CCD camera, with image stacks $(0.5-\mu \mathrm{m}$ steps) deconvolved and pertinent images of the cell $z$-projected, using AxioVision 3.1 software (Zeiss).

Kidney histology and immunofluorescence. Human kidney biopsies were fixed in Dusbosq-Brasil solution and embedded in paraffin; 2- to 6- $\mu \mathrm{m}$ sections were stained with hematoxylin-eosin, PAS, PAS methenamine silver, Masson's trichrome, and Congo red for routine diagnostics. Immunofluorescence of rat kidney sections was performed as previously described (50).

Protein purification and mass spectrometry. The E. coli ortholog of XPNPEP3, ecAPP, was cloned into the PET28a vector (Novagen) to express a fusion protein with a His tag at the $\mathrm{N}$ terminus. The resulting construct was transformed into E. coli BL21 (DE3) codon plus RIL (Stratagene). After induction by IPTG, ecAPP was purified with Ni-NTA technology (Qiagen). The 9-amino acid peptides identical to the $\mathrm{N}$-terminals of NPHP6, ALMS1, LRRC50, and dynein without methionine were synthesized by Genscript. In a typical enzymatic reaction, $100 \mu \mathrm{M}$ peptide substrate was incubated with $100 \mathrm{nM}$ purified ecAPP, which was preactivated by 20 times excess $\mathrm{MnCl}_{2}$ in $20 \mathrm{mM}$ Tris-HCl, $\mathrm{pH} 7.4,0.75 \mathrm{mM}$ $\mathrm{MnCl}_{2}$ ). The reaction was stopped by boiling samples at $100^{\circ} \mathrm{C}$ for 
5 minutes, and product was detected by ESI-LC-MS (Agilent Technologies). Candidate cleavage substrates were identified from the Ciliary proteome database (http://ciliaproteome.org).

Rescue of gastrulation phenotypes in zebrafish. MOs (Gene Tools) targeting the Danio rerio ortholog of XPNPEP3 or LRRC50 were resuspended to appropriate concentrations, using sterile deionized water, and injected into WT embryos at the 2-cell stage. To rescue mid-somite morphant phenotypes, we generated capped mRNA for MO coinjections, using linearized PCS2+ XPNPEP3 (full length or $\triangle \mathrm{N}-X P N P E P 3$ ) or PCS2 + LRRC50 (WT or mutants) as a template to transcribe mRNA with the SP6 mMessage mMachine kit (Ambion). xpпрер 3 rescue studies were carried out with 4 ng translation blocker $\mathrm{MO}$ and $50 \mathrm{pg}$ mRNA. For $\operatorname{lrc} 50$ rescue studies, $4.5 \mathrm{ng}$ of $\operatorname{lrc} 50$ translation blocker MO was coinjected with 150 pg of RNA. Gastrulation phenotypes were scored at the 10 somite stage.

Statistics. To evaluate zebrafish knockdown phenotypes, Pearson $\chi^{2}$ test was used; $P$ values of less than 0.05 were considered significant (Figure 6). Putative ciliary targets of XPNPEP3 were ranked using expectant values (Supplemental Table 8).

\section{Acknowledgments}

We sincerely thank the patients and their families for participation. We acknowledge R.H. Lyons for excellent large-scale sequencing and Jun Zhong for technical help with mass spectrometry analysis. We are grateful to the following colleagues for the contribution of helpful discussion regarding other animal models and biological systems: Jan Smeitinck (Nijmegen, Netherlands), Dontscho Kerjaschki (Vienna, Austria), Mark Winey and Chandra Kilburn (University of Colorado), Keith Gull (Oxford, United Kingdom), and Michel Leroux (Burnaby, Canada). This research was supported by grants from the NIH (DK1069274, DK1068306, and DK064614 to F. Hildebrandt; R01HD04260, R01DK072301, and R01DK075972 to N. Katsanis; DK079541 to E.E. Davis), the Macular Vision Research Foundation (to N. Katsanis), and the Foundation for Fighting Blindness (to N. Katsanis) and by a NRSA fellowship F32 DK079541 (to E.E. Davis) from the National Institute of Diabetes, Digestive and Kidney Disorders. F. Hildebrandt is an Investigator of the Howard Hughes Medical Institute, a Doris Duke Distinguished Clinical Scientist, and a Frederick G. L. Huetwell Professor. H. Khanna was supported by NIH grant EY007961 and by a grant from the Foundation for Fighting Blindness. L.M. Quarmby was supported by the Canadian Institutes for Health and Research (MOP-378061). J.F. O'Toole was supported by a grant from the NIH (DK071108). J. Uusimaa was supported by the Finnish Medical Foundation, the Alma and K.A. Snellman Foundation, and the Sigrid Juselius Foundation. This work was also supported by the Bundesministerium für Bildung und Forschung (BMBF) funded German Network for Mitochondrial Disorders (mitoNET 01GM0862) and Systems Biology of Metabotypes (SysMBo 0315494A).

Received for publication June 4, 2009, and accepted in revised form January 6, 2010.

Address correspondence to: Friedhelm Hildebrandt, Howard Hughes Medical Institute, Department of Pediatrics, University of Michigan Health System, 8220C MSRB III, 1150 West Medical Center Drive, Ann Arbor, MI 48109-5646. Phone: 734.615.7285; Fax: 734.615.1386; E-mail: fhilde@umich.edu. Or to: Nicholas Katsanis, Center for Human Disease Modeling, Department of Cell Biology, Duke University Medical Center, Durham, NC 27710. Phone: 919.613.4694; Fax: 919.684.1627; E-mail: katsanis@cellbio.duke.edu.
1. Hildebrandt F, Attanasio M, Otto E. Nephronophthisis: disease mechanisms of a ciliopathy. J Am Soc Nephrol. 2009;20(1):23-35.

2. Hildebrandt F, Zhou W. Nephronophthisisassociated ciliopathies. J Am Soc Nephrol. 2007; 18(6):1855-1871

3. Hildebrandt $\mathrm{F}$, et al. A novel gene encoding an SH3 domain protein is mutated in nephronophthisis type 1. Nat Genet. 1997;17(2):149-153.

4. Saunier $S$, et al. A novel gene that encodes a protein with a putative src homology 3 domain is a candidate gene for familial juvenile nephronophthisis. Hum Mol Genet. 1997;6(13):2317-2323.

5. Otto EA, et al. Mutations in INVS encoding inversin cause nephronophthisis type 2, linking renal cystic disease to the function of primary cilia and left-right axis determination. Nat Genet. 2003;34(4):413-420.

6. Olbrich $\mathrm{H}$, et al. Mutations in a novel gene, NPHP3, cause adolescent nephronophthisis, tapeto-retinal degeneration and hepatic fibrosis. Nat Genet. 2003;34(4):455-459.

7. Otto E, et al. A gene mutated in nephronophthisis and retinitis pigmentosa encodes a novel protein, nephroretinin, conserved in evolution. Am J Hum Genet. 2002;71(5):1167-1171.

8. Otto EA, et al. Nephrocystin-5, a ciliary IQ domain protein, is mutated in Senior-Loken syndrome and interacts with RPGR and calmodulin. Nat Genet. 2005;37(3):282-288.

9. Sayer JA, et al. The centrosomal protein nephrocystin- 6 is mutated in Joubert syndrome and activates transcription factor ATF4. Nat Genet. 2006;38(6):674-681.

10. Attanasio $M$, et al. Loss of GLIS2 causes nephronophthisis in humans and mice by increased apoptosis and fibrosis. Nat Genet.
2007;39(8):1018-1024

11. Delous M, et al. The ciliary gene RPGRIP1L is mutated in cerebello-oculo-renal syndrome (Joubert syndrome type B) and Meckel syndrome. Nat Genet. 2007;39(7):875-881.

12. Otto EA, Trapp ML, Schultheiss UT, Helou J, Quarmby LM, Hildebrandt F. NEK8 mutations affect ciliary and centrosomal localization and may cause nephronophthisis. J Am Soc Nephrol. 2008;19(3):587-592.

13. Otto EA, et al. Hypomorphic mutations in meckelin (MKS3/TMEM67) cause nephronophthisis with liver fibrosis (NPHP11). J Med Genet. 2009;46(10):663-670.

14. Watnick T, Germino G. From cilia to cyst. Nat Genet. 2003;34(4):355-356.

15. Badano JL, Mitsuma N, Beales PL, Katsanis N. The ciliopathies: an emerging class of human genetic disorders. Annu Rev Genomics Hum Genet. 2006;7:125-148.

16. Nauli SM, et al. Polycystins 1 and 2 mediate mechanosensation in the primary cilium of kidney cells. Nat Genet. 2003;33(2):129-137.

17. Fischer E, et al. Defective planar cell polarity in polycystic kidney disease. Nat Genet. 2006;38(1):21-23.

18. Simons $M$, et al. Inversin, the gene product mutated in nephronophthisis type II, functions as a molecular switch between Wnt signaling pathways. Nat Genet. 2005;37(5):537-543.

19. Ma Z, et al. Mutations of HNF-1beta inhibit epithelial morphogenesis through dysregulation of SOCS-3. Proc Natl Acad Sci U S A. 2007;104(51):20386-20391.

20. Saburi S, et al. Loss of Fat 4 disrupts PCP signaling and oriented cell division and leads to cystic kidney disease. Nat Genet. 2008;40(8):1010-1015.

21. Kishimoto N, Cao Y, Park A, Sun Z. Cystic kidney gene seahorse regulates cilia-mediated processes and Wnt pathways. Dev Cell. 2008;14(6):954-961.

22. Gerdes JM, et al. Disruption of the basal body compromises proteasomal function and perturbs intracellular Wnt response. Nat Genet. 2007;39(11):1350-1360.

23. Khanna $\mathrm{H}$, et al. A common allele in RPGRIP1L is a modifier of retinal degeneration in ciliopathies. Nat Genet. 2009;41(6):739-745.

24. den Hollander AI, et al. Mutations in the CEP290 (NPHP6) gene are a frequent cause of Leber congenital amaurosis. Am J Hum Genet. 2006;79(3):556-561.

25. Leitch CC, et al. Hypomorphic mutations in syndromic encephalocele genes are associated with Bardet-Biedl syndrome. Nat Genet. 2008;40(4):443-448.

26. Valente EM, et al. Mutations in CEP290, which encodes a centrosomal protein, cause pleiotropic forms of Joubert syndrome. Nat Genet. 2006;38(6):623-625.

27. Baala L, et al. Pleiotropic effects of CEP290 (NPHP6) mutations extend to Meckel syndrome. Am J Hum Genet. 2007;81(1):170-179.

28. Gherman A, Davis EE, Katsanis N. The ciliary proteome database: an integrated community resource for the genetic and functional dissection of cilia. Nat Genet. 2006;38(9):961-962.

29. Hoskins BE, Thorn A, Scambler PJ, Beales PL. Evaluation of multiplex capillary heteroduplex analysis: a rapid and sensitive mutation screening technique. Hum Mutat. 2003;22(2):151-157.

30. Otto EA, et al. Mutation analysis in nephronophthisis using a combined approach of homozygosity mapping, CEL I endonuclease cleavage, and direct sequencing. Hum Mutat. 2008;29(3):418-426.

31. Ersahin C, Szpaderska AM, Orawski AT, Simmons WH. Aminopeptidase P isozyme expression in human tissues and peripheral blood 
mononuclear cell fractions. Arch Biochem Biophys. 2005;435(2):303-310.

32. Graham SC, Bond CS, Freeman HC, Guss JM. Structural and functional implications of metal ion selection in aminopeptidase P, a metalloprotease with a dinuclear metal center. Biochemistry. 2005;44(42):13820-13836.

33. Li X, et al. Structure of human cytosolic X-prolyl aminopeptidase: a double Mn(II)-dependent dimeric enzyme with a novel three-domain subunit. J Biol Chem. 2008;283(33):22858-22866.

34. Yoshimoto $\mathrm{T}$, Murayama N, Honda T, Tone $\mathrm{H}$, Tsuru D. Cloning and expression of aminopeptidase P gene from Escherichia coli HB101 and characterization of expressed enzyme. J Biochem. 1988;104(1):93-97.

35. Ross AJ, et al. Disruption of Bardet-Biedl syndrome ciliary proteins perturbs planar cell polarity in vertebrates. Nat Genet. 2005;37(10):1135-1140.

36. Li G, et al. A role for Alstrom syndrome protein, alms 1 , in kidney ciliogenesis and cellular quiescence. PLoS Genet. 2007;3(1):e8.

37. Sullivan-Brown J, et al. Zebrafish mutations affecting cilia motility share similar cystic phe- notypes and suggest a mechanism of cyst formation that differs from pkd2 morphants. Dev Biol. 2008;314(2):261-275.

38. Badano JL, et al. Dissection of epistasis in oligogenic Bardet-Biedl syndrome. Nature. 2006;439(7074):326-330.

39. Corbit KC, Aanstad P, Singla V, Norman AR Stainier DY, Reiter JF. Vertebrate Smoothened functions at the primary cilium. Nature. 2005;437(7061):1018-1021.

40. Corbit KC, et al. Kif3a constrains beta-catenindependent Wnt signalling through dual ciliary and non-ciliary mechanisms. Nat Cell Biol. 2008;10(1):70-76.

41. Follit JA, Tuft RA, Fogarty KE, Pazour GJ. The intraflagellar transport protein IFT2 0 is associated with the Golgi complex and is required for cilia assembly. Mol Biol Cell. 2006;17(9):3781-3792.

42. Omran H, et al. Ktu/PF13 is required for cytoplasmic pre-assembly of axonemal dyneins. Nature. 2008;456(7222):611-616.

43. Hildebrandt F, et al. A systematic approach to mapping recessive disease genes in individuals from outbred populations. PLoS Genet. 2009;5(1):e1000353.
44. Meisinger C, et al. A genome-wide association study identifies three loci associated with mean platelet volume. Am J Hum Genet. 2009;84(1):66-71.

45. Torres JZ, Miller JJ, Jackson PK. High-throughput generation of tagged stable cell lines for proteomic analysis. Proteomics. 2009;9(10):2888-2891.

46. Janssen AJ, et al. Spectrophotometric assay for complex I of the respiratory chain in tissue samples and cultured fibroblasts. Clin Chem. 2007;53(4):729-734.

47. Janssen AJ, et al. Measurement of the energy-generating capacity of human muscle mitochondria: diagnostic procedure and application to human pathology. Clin Chem. 2006;52(5):860-871.

48. Rasmussen HN, Andersen AJ, Rasmussen UF. Optimization of preparation of mitochondria from 25-100 mg skeletal muscle. Anal Biochem. 1997;252(1):153-159.

49. Rustin $P$, et al. Biochemical and molecular investigations in respiratory chain deficiencies. Clin Chim Acta. 1994;228(1):35-51.

50. Hinkes B, et al. Positional cloning uncovers mutations in PLCE1 responsible for a nephrotic syndrome variant that may be reversible. Nat Genet. 2006;38(12):1397-1405. 